\title{
Effect of ceramic thickness, grinding, and aging on the mechanical behavior of a polycrystalline zirconia
}

\section{Rodrigo Diniz PRADO(a) Gabriel Kalil Rocha PEREIRA(b) Marco Antonio BOTTINO(a) Renata Marques de MELO(a) Luiz Felipe VALANDRO(b)}

(a) Universidade Estadual Paulista - Unesp, Dental School, Prosthodontics Units, São José dos Campos, SP, Brazil.

(b) Universidade Federal de Santa Maria - UFSM, Faculty of Odontology, Prosthodontics Units, Santa Maria, RS, Brazil.

Declaration of Interest: The authors certify that they have no commercial or associative interest that represents a conflict of interest in connection with the manuscript.

\section{Corresponding Author:}

Luiz Felipe Valandro

Ifvalandro@hotmail.com

https://doi.org/10.1590/1807-3107BOR-2017.vol31.0082

Submitted: Apr 26, 2017

Accepted for publication: July 27, 2017

Last revision: Aug 14, 2017

\begin{abstract}
Monolithic restorations of Y-TZP have been recommended as a restorative alternative on prosthetic dentistry as it allows a substantial reduction of ceramic thickness, which means a greater preservation of tooth structure. However, the influence of grinding and aging when using a thinner layer of the material is unclear. This investigation aimed to evaluate and compare the effects of ceramic thickness $(0.5 \mathrm{~mm}$ and $1.0 \mathrm{~mm}$ ), grinding and aging (low-temperature degradation) on the mechanical behavior and surface characteristics of a full-contour Y-TZP ceramic. Y-TZP disc-shaped specimens (15 mm diameter) were manufactured with both thicknesses and randomly assigned into 4 groups considering the factors 'grinding with diamond bur' and 'aging in autoclave'. Surface topography (roughness, 3D profilometry and SEM), phase transformation, flexural strength and structural reliability (Weibull) analyses were executed. Grinding affected the surface topography, while aging did not promote any effect. An increase in $m$-phase content was observed after grinding and aging, although different susceptibilities were observed. Regardless of zirconia's thickness, no deleterious effect of grinding or aging on the mechanical properties was observed. Thus, in our testing assembly, reducing the thickness of the Y-TZP ceramic did not alter its response to grinding and low temperature degradation and did not impair its mechanical performance.
\end{abstract}

Keywords: Prosthodontics; Ceramics.

\section{Introduction}

A major advantage of Yttrium-stabilized Tetragonal Zirconia Polycrystal (Y-TZP) is its high fracture resistance, having a flexural strength of 900-1000 MPa and fracture toughness of 5.5 to $7.4 \mathrm{MPa} / \mathrm{m}^{2}$. These properties are very important and demonstrate an enhanced ability to resist to the occlusal forces generated during chewing, when compared with lithium disilicate ceramic, for example, which has a flexural strength of $350 \mathrm{MPa}$ and a fracture toughness of $3.2 \mathrm{MPa} / \mathrm{m}^{2}$.

Hence, zirconia-based materials are the most resistant among the existing dental ceramics - they are used in the manufacturing of frameworks of single or multi-unit FPDs (fixed partial dentures), and recently, for monolithic restorations. ${ }^{3,4}$ The major advantage of monolithic restorations is that they allow a substantial reduction of ceramic thickness by eliminating 
the veneer layer without compromising the final strength of the system. Thus, this assembly results in a more conservative tooth preparation and decreased removal of tooth structure. ${ }^{3,5}$ Besides, clinical studies have shown the fracture or chipping of the veneering porcelain as the main complication for FPDs, another advantage of using monolithic restorations. ${ }^{1}$

Zirconia is a polymorphic metastable material, naturally occurring in three different structural forms (monoclinic $-m$; tetragonal $-t$; and cubic $-c$ ). Without the addition of stabilizers, such as 3\% mol of yttrium oxide (stabilized zirconia at $t$-phase form), pure zirconia does not present the stability required for use in biomedical fields. However, even with the addition of stabilizers, phase transformation ( $t$ to $m$ phase) still may take place in response of mechanical, physical and/or chemical stimuli. ${ }^{6,7}$

The $t-m$ phase transformation causes a local volumetric expansion of approximately $3-4 \%$, resulting in surface compressive stress concentration that, when located near surface defects/cracks, will act as a defense mechanism to prevent crack propagation. ${ }^{8,9}$ Firstly, this phase transformation mechanism occurs on superficial grains, with water incorporation and filling of the oxygen spaces. Subsequently, it spreads, resulting in rougher surface, and a zirconia with decreased hardness, fracture toughness and density, ${ }^{7}$ which is known as low-temperature degradation. ${ }^{10}$

As previously mentioned, monolithic restorations allow a substantial reduction on material thickness, allowing a more conservative preparation and resulting in greater preservation of the tooth structure, reducing the risk of injury to the pulp and of trauma. ${ }^{11}$ However, there is scarce information in the literature regarding the influence of adjustments (to enhance occlusal relations, fit and emergence profile) and aging when thinner layers of the material are considered. Nakamura et al..$^{5}$ showed that with a reduction on occlusal thickness the load to fracture is also reduced. Therefore, the raised question is: would a reduced Y-TZP ceramic thickness lead to a higher susceptibility of defects due to grinding and aging, and affect the mechanical behavior?

Thus, the present study aimed to evaluate the influence of ceramic thickness, grinding with diamond bur and aging in autoclave on the flexural strength, structural reliability, topography and phase transformation of a zirconia indicated for monolithic restoration. The study hypothesis was "a reduced ceramic thickness will predispose to an impairment on mechanical performance after grinding and aging".

\section{Methods}

\section{Sample preparation}

Disc-shaped specimens were manufactured according to ISO $6872-2008^{12}$ for biaxial flexure strength testing of ceramic materials (final dimensions of $15 \mathrm{~mm}$ in diameter), using the methodology previously described in the literature. ${ }^{13,14,15}$ Basically, pre-sintered blocks of Y-TZP (Lot no. 637590 Rev.0 2011-03, Zirlux FC, Ardent Dental, Inc, Amherst, NY, USA - which present approximately $20 \%$ of shrinkage, as declared by the manufacturer) were ground into cylinders of 18 mm diameter using 600-1200 grit silicon carbide ( $\mathrm{SiC}$ ) paper (3M, St Paul, MN, USA) under water-cooling. The cylinders were cross-sectioned using a precision saw machine (ISOMET 1000, Buehler, Lake Bluff, IL) and polished with $\mathrm{SiC}$ papers (600-1200 grit) to produce disc-shaped samples with pre-sintering thickness of approximately $0.6 \mathrm{~mm}$ and $1.2 \mathrm{~mm}$. Then the ceramic samples were sintered as recommended by the manufacturer's guidelines, having final dimensions of $15 \mathrm{~mm}$ in diameter and 0.5 or $1 \mathrm{~mm}$ in thickness. Finally, the specimens of both thicknesses were randomly divided into 4 groups $(n=30)$, considering the factors "grinding" and "aging (low-temperature degradation)", as it shows in Table 1.

\section{Surface Treatment}

Samples from the control group (C) remained untouched after the sintering process - "as-sintered" samples.

\section{Grinding}

Grinding was performed by a single trained operator following the protocol recommended on the systematic review by Pereira et al. ${ }^{16}$ Extra-fine diamond burs (\#3101FF - grit size 30 m; KG Sorensen, Cotia, Brazil) coupled to a contra-angle handpiece (T2 REVO R170 contra-angle handpiece of 170,000 rpm, Sirona, Bensheim, Germany) combined with a low-speed motor 
Table 1. Experimental design.

\begin{tabular}{|c|c|c|c|c|}
\hline Thickness (mm) & Surface treatment & $\begin{array}{l}\text { Low-temperature aging } \\
\text { in autoclave }\end{array}$ & $\begin{array}{l}\text { Group } \\
\text { code }\end{array}$ & $\begin{array}{l}\text { Sample size } \\
(n)\end{array}$ \\
\hline \multirow{4}{*}{0.5} & \multirow{2}{*}{ Control, as-sintered (without any additional treatment) } & No & C & 30 \\
\hline & & Yes & C-Ltd & 30 \\
\hline & \multirow{2}{*}{$\begin{array}{l}\text { Grinding with extra-fine diamond bur (\#3101FF - } 30 \mu \mathrm{m} \\
\text { grit size; KG Sorensen, Cotia, Brazil) }\end{array}$} & No & G & 30 \\
\hline & & Yes & G-Ltd & 30 \\
\hline \multirow{4}{*}{1.0} & \multirow{2}{*}{ Control, as-sintered (without any additional treatment) } & No & C & 30 \\
\hline & & Yes & C-Ltd & 30 \\
\hline & \multirow{2}{*}{$\begin{array}{l}\text { Grinding with extra-fine diamond bur (\#3101FF - grit size } \\
\qquad 30 \mu \mathrm{m} ; \mathrm{KG} \text { Sorensen, Cotia, Brazil) }\end{array}$} & No & G & 30 \\
\hline & & Yes & G-Ltd & 30 \\
\hline
\end{tabular}

(Kavo Dental, Biberach, Germany) under constant water-cooling ( $\approx 30 \mathrm{ml} / \mathrm{min}$ ) were used; the diamond bur was replaced after each specimen. Caution was taken to increase reproducibility and standardize wear thickness during grinding, following the grinding protocol previously described in the literature. , $^{14,17,18}$

\section{Low-temperature aging}

Low-Temperature Degradation (LTD) was simulated in an autoclave (Sercon HS1-0300 $\mathrm{n}^{\circ} 1560389 / 1$ ) at $134^{\circ} \mathrm{C}$, under $2 \mathrm{bar}$, over a period of $20 \mathrm{~h}$, following the protocol suggested on the systematic review by Pereira et al. ${ }^{19}$

\section{Surface analysis (Topography, Roughness and Phase transformation) SEM analysis and 3D optical profilometer analysis}

The superficial topography was accessed using scanning electron microscope (JSM-6360, JEOL, Tokyo, Japan). For that, the specimens $(n=2)$ were coated with a gold-palladium alloy spray and inspected to evaluate the surface pattern.

Additional analysis $(n=2)$ was performed under 3D optical digital profilometer (Wyko, NT 1100, Veeco, EUA). which was connected to a computer using the software Wyko Vison 32 (Veeco, EUA).

\section{Roughness analysis}

Surface roughness analysis was performed for all specimens in all conditions ( $\mathrm{n}=30$, Mitutoyo SJ-410, Mitutoyo Corporation, Takatsu-ku, Kawasaki, Kanagawa, Japan): three measurements were made for each specimen according to the ISO 1997 parameters (Ra - arithmetical mean of the absolute values of peaks and valleys measured from a medium plane (mm) and $\mathrm{Rz}$ - average distance between the five highest peaks and five major valleys found in the standard (mm)) with a cut-off ( $\mathrm{n}=5), \lambda C 0.8 \mathrm{~mm}$ and $\lambda S 2.5 \mu \mathrm{m}$. Mean values of all measurements from each specimen were then obtained.

\section{Phase analysis by X-ray diffraction (XRD Analysis)}

Quantitative analysis of phase transformation was conducted $(n=3)$ to determine the relative amount of $\mathrm{m}$-phase and depth of the transformed layer under each condition. The analysis was performed using an X-ray diffractometer (Bruker AXS, D8 Advance, Karlsruhe, Germany) with a $\mathrm{CuK} \alpha$ radiation. Spectra were collected using the Bragg-Brentano geometry in the $2 \theta$ range from 25 to 35 degrees, at a step interval of $1 \mathrm{~s}$, and step size of 0.03 degrees/step.

The amount of $m$-phase was calculated using the method introduced by Garvie and Nicholson ${ }^{20}$ modified by Toraya et al. ${ }^{21}$ This method has been extensively used and described in the literature, as found in the systematic review by Pereira et al. ${ }^{16}$

The depth of the transformed layer was calculated based on the amount of the $m$-phase, considering that a constant fraction of grains had symmetrically transformed to the $m$-phase along the surface, as recommended by Kosmac et al. ${ }^{22}$

\section{Biaxial flexure test}

Specimens $(n=30)$ were subjected to a biaxial flexural strength test according to ISO 6872-2008, ${ }^{12}$ as previously described..$^{13,14,15}$ Specimens were placed on three support balls $(\varnothing 3.2 \mathrm{~mm})$ positioned equidistant from each other, with the treated surface facing down (tensile stress concentration area), under water; a circular tungsten flat piston $(\varnothing=1.6 \mathrm{~mm})$ was used to apply the 
load (1 $\mathrm{mm} / \mathrm{min})$ with a universal testing machine (EMIC DL-1000, EMIC, São José dos Pinhais, Brazil) until the catastrophic failure of the material. A $1000 \mathrm{kgf}$ load was applied to the disc center. A covering tape was positioned where the load was applied to prevent the dispersal of fragments ${ }^{23}$ and to promote better contact between the piston and the specimen. ${ }^{24}$

\section{Data analyses}

A descriptive analysis of the roughness data (Ra and Rz) was performed in Minitab 16 program (Minitab 17.1.0, Minitab, Inc, USA) to determine the mean and standard deviations. As data presented a parametric and homogeneous distribution (using Shapiro-Wilk and Levene tests, respectively), the two-way ANOVA and the post-hoc Tukey's test were performed considering the two factors (grinding and aging) and the interaction between them. Additionally, a Pearson correlation analysis was made with the roughness $\mathrm{Ra}$ and the biaxial strength parameters.

The Weibull statistical analysis was carried out to describe the structural homogeneity of the ceramic material, which is a way to describe the resistance variation ${ }^{25}$. Thus, the modulus (m) and the Weibull characteristic strength $(\sigma c)$ were obtained with $95 \%$ confidence interval as determined in the diagram (in accordance with DIN ENV 843-526). The characteristic strength is the strength at a failure probability of approximately $63 \%$, and the Weibull modulus is used as a measure of the distribution of strengths, expressing the reliability of the material.

\section{Results}

3D Profilometer images and SEM micrographs showed that grinding produced an alteration of surface pattern, with multi-directional scratches following the movement of the diamond bur, while the "as sintered" group (control) showed a homogeneous surface (Figure 1). Aging did not cause any relevant alteration of those patterns.

Roughness analysis showed that, for both Ra and $\mathrm{Rz}$ parameters, the surface treatment presented a statistical difference $(p<0.05)$, while aging (Ra: $p=0.348$; Rz: $p=0.119$ ) and the interaction of factors (Ra: $p=0.337 ; \mathrm{Rz}: \mathrm{p}=0.407)$ did not. Grinding with diamond burs led to statistically significant higher roughness values ( $\mathrm{Ra}$ and $\mathrm{Rz}$ parameters) in comparison to absence of grinding $(p<0.05)$. Additionally, aging in autoclave (LTD) did not lead to any impact on roughness (Table 2). Regarding the Pearson correlation test between Ra and biaxial strength, a very weak positive statistical correlation was observed $(p=0.280)$.

The low temperature regimen promoted an increase in $m$-phase content, which was more intense for the control group (58.17\%). Grinding also increased the $m$-phase content, although it led to a decrease in the material susceptibility to new phase transformations during aging (Figure 2).

Independent of zirconia's thickness, the lowtemperature aging and grinding led to an increase in characteristic strengths, when compared to control groups (Table 2). Thus, the reduced thickness (0.5 $\mathrm{mm}$ ) did not promote lower characteristic strength compared with thicker specimens (Table 2). The lowtemperature aging and grinding had no influence on Weibull modulus ( $m$ value) of the groups with different zirconia thickness (i.e. neither grinding with diamond bur nor the aging protocol significantly reduced the material reliability).

\section{Discussion}

The current study found that grinding and low-temperature aging in autoclave did not promote a negative effect on the characteristic strength and structural reliability (Weibull moduli) of the material, for both Y-TZP thicknesses $(0.5$ and $1 \mathrm{~mm})$. Thus, the hypothesis that a reduced Y-TZP ceramic thickness would increase impairment on mechanical performance after grinding and aging was rejected.

3D optical profilometry and SEM images showed an irregular surface pattern with many peaks and valleys due to grinding with diamond bur. Additionally, XRD analysis showed that grinding leads to $m$-phase content increase (approximately 9\% of $m$-phase content as noticed on Figure 2). Consequently, ground specimens showed higher values of characteristic strength and this can be explained by the transformation toughening mechanism counterbalancing any potential critical defect introduced by grinding 9 . 


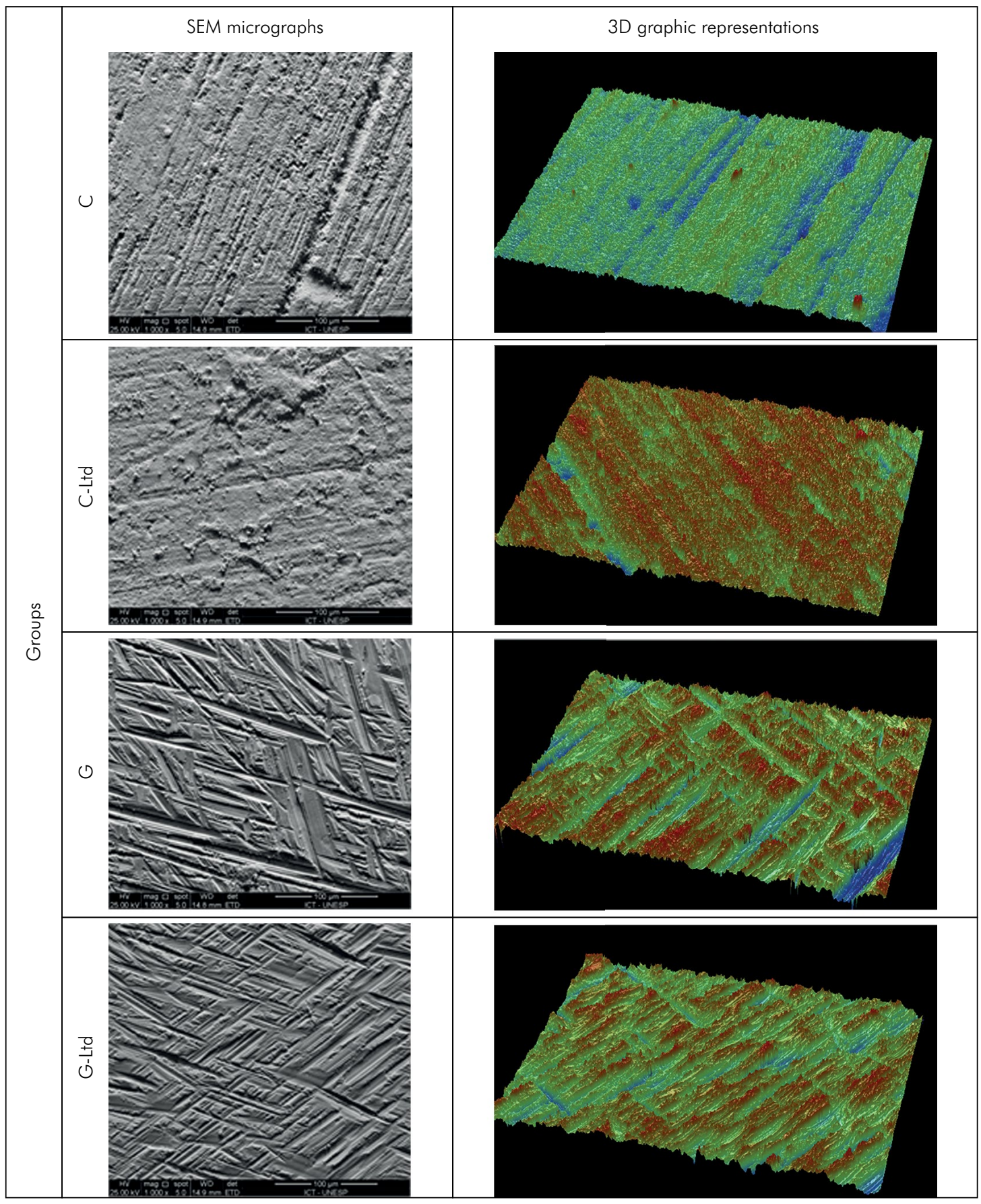

Figure 1. SEM micrographs (1000 x magnification) and 3D graphic representations showing different topographical patterns of specimens subjected to studied conditions. It highlights that both evaluated ceramic thicknesses $(0.5 \mathrm{and} 1 \mathrm{~mm})$ presented the same topographical pattern and that grinding procedure affects the materials topography changing its superficial pattern, while low temperature aging appears to not interfere in such outcome (C-as-sintered; G-grinding; Ltd-low-temperature degradation)

When a crack initiates and propagates towards the bulk of the material, tensile stress concentration at the crack tip leads the surrounding area to a monoclinic phase transformation (responsible for a $\sim 4 \%$ volumetric expansion). The crack becomes surrounded by transformed zirconia, producing a compressive stress in this zone, making crack propagation more difficult. $8,9,15$ 
Thus, even though the groups subjected to grinding procedure presented higher values of roughness (Ra and Rz), it did not have a negative impact on their characteristic strength. Another potential explanation for that might be related to the grinding protocol used by us (diamond bur of $30-\mu \mathrm{m}$ grit size, low-speed motor coupled to a contra-angle handpiece, capable to multiply the velocity and watercooling). This allows a better control of the grinding procedure (in comparison to normal high speed handpieces), producing less defects and triggering the transformation toughening mechanism. ${ }^{14,16,27}$

These findings are not in agreement with previous literature, ${ }^{28,29,30,31}$ in which grinding with diamond burs did not promote an increase in $m$-phase content and created severe defects on the material surface leading to degradation of the mechanical properties. A high-speed handpiece used by those authors can lead to a temperature increase at the Y-TZP surface, consequently triggering a reverse $m$ - $t$ transformation that works against the transformation toughening mechanism. ${ }^{30,31,32}$

From that viewpoint, the presence of abundant water cooling and the use of a gentle grinding protocol seems to contribute to the maintenance of the mechanical properties of the material ${ }^{16}$, whereas grinding without proper cooling or with an aggressive protocol may raise the surface temperature ${ }^{30,31,32}$ to the critical point where $t-m$ phase transformation occurs. Consequently, more defects are produced to the material and the occurrence of the transformation toughening mechanism is prevented, resulting in decrease of the Y-TZP strength. . $8,29,30,31,33,34,35^{2}$

Our data shows that surface roughness should not be solely used to predict the mechanical behavior of Y-TZP ceramics, since the $t-m$ phase transformation (increase in $m$-phase content) may counterbalance any potential defect inflicted and lead to a final increase
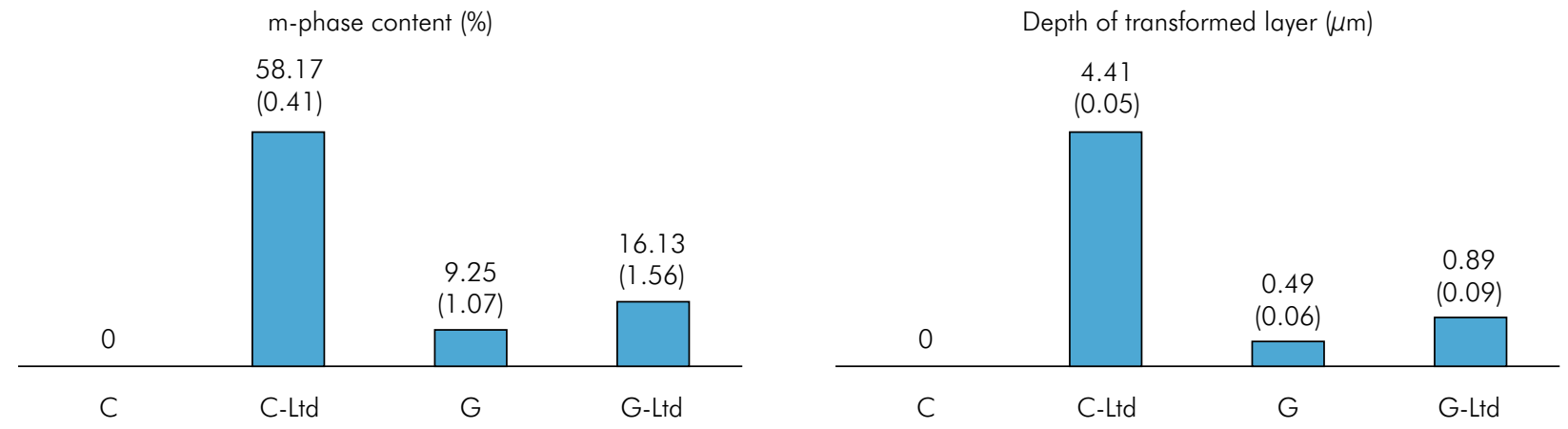

Figure 2. Mean percentage of monoclinic phase content (\%) of the Y-TZP surface and depth of transformed layer ( $\mu \mathrm{m}$ ), in addition to their respective standard deviations (data inside parentheses). It highlights the highest $\mathrm{m}$-phase content at as-sintered after aging condition (C-Ltd), as well as, the deepest layer of phase transformation $(\mu \mathrm{m})$. Grinding procedure appears to trigger an initial m-phase content increase, although it prevents new phase transformation during aging (C-as-sintered; $G$-grinding; Ltd- low-temperature degradation).

Table 2. Analysis of variance (two-way ANOVA) of roughness data (Ra and Rz), characteristic strength ( $\left.\sigma_{c}\right)$, Weibull modulus $(m)$ and respective confidence intervals $(95 \% \mathrm{Cl})$ for treatment and aging factors.

\begin{tabular}{lccccccc}
\hline \multirow{2}{*}{$\begin{array}{l}\text { Thickness } \\
(\mathrm{mm})\end{array}$} & Groups & \multicolumn{2}{c}{ Roughness $(\mu \mathrm{m})$} & \multicolumn{3}{c}{ Weibull analysis } \\
\cline { 3 - 8 } & $\mathrm{Ra}$ & $\mathrm{Rz}$ & $\sigma_{\mathrm{c}}$ & $95 \% \mathrm{Cl}$ & $\mathrm{m}$ & $95 \% \mathrm{Cl}$ \\
\hline & $\mathrm{C}$ & $0.4(0.1)^{\mathrm{C}}$ & $3.4(0.8)^{\mathrm{BC}}$ & $726.9^{\mathrm{A}}$ & $688.74-766.00$ & $8.1^{\mathrm{AB}}$ & $6.13-10.63$ \\
0.5 & $\mathrm{C}-\mathrm{Ltd}$ & $0.4(0.1)^{\mathrm{C}}$ & $3.1(1.0)^{\mathrm{C}}$ & $1183.3^{\mathrm{D}}$ & $1087.26-1284.78$ & $5.1^{\mathrm{A}}$ & $3.14-8.40$ \\
& $\mathrm{G}$ & $0.6(0.2)^{\mathrm{A}}$ & $4,0(0.8)^{\mathrm{AB}}$ & $986.3^{\mathrm{BC}}$ & $923.98-1051.02$ & $6.7^{\mathrm{AB}}$ & $4.67-9.48$ \\
& $\mathrm{G}-\mathrm{Ltd}$ & $0.5(0.1)^{\mathrm{AB}}$ & $3.6(0.7)^{\mathrm{ABC}}$ & $1162.3^{\mathrm{D}}$ & $1120.07-1204.92$ & $11.8^{\mathrm{B}}$ & $9.41-14.65$ \\
& $\mathrm{C}$ & $0.4(0.2)^{\mathrm{BC}}$ & $3.6(0.9)^{\mathrm{ABC}}$ & $760.7^{\mathrm{A}}$ & $725.36-796.75$ & $9.1^{\mathrm{AB}}$ & $7.02-11.87$ \\
1.0 & $\mathrm{C}-\mathrm{Ltd}$ & $0.5(0.2)^{\mathrm{BC}}$ & $3.7(1.0)^{\mathrm{ABC}}$ & $1023.9^{\mathrm{C}}$ & $972.83-1076.15$ & $8.5^{\mathrm{AB}}$ & $6.65-10.85$ \\
& $\mathrm{G}$ & $0.6(0.1)^{\mathrm{A}}$ & $4.0(0.8)^{\mathrm{A}}$ & $907.1^{\mathrm{B}}$ & $861.73-953.59$ & $8.5^{\mathrm{AB}}$ & $6.07-11.80$ \\
& $\mathrm{G}-\mathrm{Ltd}$ & $0.6(0.1)^{\mathrm{A}}$ & $4.0(0.7)^{\mathrm{AB}}$ & $1027.7^{\mathrm{C}}$ & $971.26-1085.75$ & $7.7^{\mathrm{AB}}$ & $5.31-11.15$ \\
\hline
\end{tabular}


in the material mechanical performance, which is also demonstrated by previous in vitro studies. ${ }^{14,17,33}$

Therefore, the surface modifications due to grinding are able to cause positive or negative effects on the mechanical properties of Y-TZP materials. If the defect produced by grinding has a greater depth than the one of the compressive layer created by $t-m$ phase transformation, it may result in higher levels of tensile stress concentration, raising the incidence of catastrophic failures. ${ }^{28,30,36}$ However, when these defects have a smaller depth than the one of the compressive stress layer (created by transformation toughening mechanism), the crack propagation and catastrophic failures are avoided by the surrounding compressive stresses. .,37 $^{2}$

Regarding aging, the as-sintered condition showed an extensive increase in $\sigma_{c}$ after the aging protocol, and this result is probably due to an intense increase in monoclinic phase content $(58.17 \%)$ leading to transformation, and contributing to the increase in toughening. It is important to note that even at smaller thickness $(0.5 \mathrm{~mm})$ this monoclinic phase content did not lead to any deleterious influence on $\sigma_{c^{\prime}}$ which corroborates the assertion that this material can be used even with reduced thickness.

The different values of $m$-phase for the as-sintered group and grinding groups after aging (C-Ltd $58.17 \%$, G-Ltd - 16.13\%) support another important fact: ground surfaces seem to be less susceptible to $t-m$ phase transformation during aging, which is probably caused by a protective effect achieved by formation of the tension barrier by $t-m$ transformation on the surface of the material. ${ }^{38}$

According to Muñoz-Tabarez et al., ${ }^{39}$ the microstructural changes induced by grinding of Y-TZP consist of three well defined layers, from the surface to the interior: a) a superficial crystallized zone, where grain diameter range from 10 to $20 \mathrm{~nm}$ approximately; b) a plastically deformed zone; and c) a zone in which tetragonal to monoclinic phase transformation take place, which is mainly responsible for the formation of compressive residual stresses that usually increases the flexure strength and apparent fracture toughness of ground specimens. ${ }^{39}$

Thus, the increased resistance to hydrothermal degradation after grinding is possibly related to the existence of this very thin layer of tetragonal recrystallized nano-grains $(10-20 \mathrm{~nm})$ that are smaller than the critical size for transformation in humid environment, in addition to the presence of residual compressive stress on the surface of Y-TZP ceramics. ${ }^{39,40}$

Although the tested Y-TZP ceramic consists of a new translucent material, and therefore alterations on microstructureand composition (that are notclearly stated by the manufacturer) may be expected in comparison to conventional Y-TZP ceramics ${ }^{4}$, our data support a similar performance of the tested ceramic to the ones that have been evaluated by previous studies. ${ }^{15,16,17,18,19}$

Finally, we showed that the protocols for grinding and aging used by this study do not promote a deleterious effect on the mechanical properties of Y-TZP ceramic, regardless of the ceramic thickness. However, more studies subjecting the Y-TZP materials to drastic environments such as fatigue tests, long periods in the presence of moisture, as well as crownshape sample testing set-ups, should be conducted to support these findings.

The test assembly recommended by ISO 6872$2008^{12}$ and used by us requires that the surface being tested be positioned directed to the tension side (where the fracture originates). Clinically, however, when doing occlusal adjustments with diamond burs on a monolithic crown, the surface subjected to grinding will be mostly under compression during chewing. Thus, this might be considered an important limitation of our study.

Although our study shows that a thinner layer (0.5 $\mathrm{mm}$ ) did not impair the mechanical performance of Y-TZP ceramic in accordance with previous in vitro studies, ${ }^{5,11}$ well-designed in vivo studies are necessary to obtain information regarding clinical performance of thin restorations, such as aesthetic potential, wear performance, as well as mechanical behavior.

\section{Conclusions}

Grinding and low temperature aging in autoclave did not affect the mechanical behavior of the tested translucent zirconia, with $0.5 \mathrm{~mm}$ and $1 \mathrm{~mm}$ thicknesses. However, those conditions led to a high $m$-phase content.

A thinner layer $(0.5 \mathrm{~mm})$ presented similar or higher characteristic strength compared with the 1.0 $\mathrm{mm}$ layer, both before and after grinding and aging. 


\section{Acknowledgements}

The authors state that there is no conflict of interest. We thank CAPES (Agency for the High-Standard Promotion

\section{References}

1. Triwatana P, Nagaviroj N, Tulapornchai C. Clinical performance and failures of zirconia-based fixed partial dentures: a review literature. J Adv Prosthodont. 2012;4(2):76-83. https://doi.org/10.4047/jap.2012.4.2.76

2. Craig RG. Restorative dental materials. 11th ed. St Louis: Mosby; 2002.

3. Denry I, Kelly JR. Emerging ceramic-based materials for dentistry. J Dent Res. 2014;93(12):1235-42. https://doi.org/10.1177/0022034514553627

4. Stawarczyk B, Frevert K, Ender A, Roos M, Sener B, Wimmer T. Comparison of four monolithic zirconia materials with conventional ones: contrast ratio, grain size, four-point flexural strength and two-body wear. J Mech Behav Biomed Mater. 2016;59:128-38. https://doi.org/10.1016/i.jmbbm.2015.11.040

5. Nakamura K, Harada A, Inagaki R, Kanno T, Niwano Y, Milleding $P$ et al. Fracture resistance of monolithic zirconia molar crowns with reduced thickness. Acta Odontol Scand. 2015;73(8):602-8. https://doi.org/10.3109/00016357.2015.1007479

6. Piconi C, Maccauro G. Zirconia as a ceramic biomaterial. Biomaterials. 1999;20(1):1-25. https://doi.org/10.1016/S0142-9612(98)00010-6

7. Chevalier J, Gremillard L, Deville S. Low-temperature degradation of zirconia and implications for biomedical implants. Annu Rev Mater Res. 2007;37(1):1-32. https://doi.org/10.1146/annurev.matsci.37.052506.084250

8. Garvie R, Hannink R, Pascoe R. Ceramic steal? Nature. 1975;258(5537):703-4. https://doi.org/10.1038/258703a0

9. Hannink R, Kelly P, Muddle B. Transformation toughening in zirconia containing ceramics. J Am Ceram Soc. 2000;83(3):461-87. https://doi.org/10.1111/j.1151-2916.2000.tb01221.x

10. Kobayashi K, Kuwajima H, Masaki T. Phase change and mechanical properties of $\mathrm{ZrO} 2-\mathrm{Y} 2 \mathrm{O} 3$ solid electrolyte after ageing. Solid State Ion. 1981;3-4:489-95. https://doi.org/10.1016/0167-2738(81)90138-7

11. Sun T, Zhou S, Lai R, Liu R, Ma S, Zhou Z et al. Load-bearing capacity and the recommended thickness of dental monolithic zirconia single crowns. J Mech Behav Biomed Mater. 2014;35:93-101. https://doi.org/10.1016/i.jmbbm.2014.03.014

12. International Organization for Standardization. ISO 6872:2015. Dentistry : ceramic materials. Geneva: International Organization for Standardizatio; 2015.

13. Amaral M, Valandro LF, Bottino MA, Souza RO. Low-temperature degradation of a Y-TZP ceramic after of Graduate Courses, Brazil) and CNPq agencies for supporting this study (scholarships) and Ivoclar Vivadent (Schaan, Liechtenstein) for the donation of ceramics.

surface treatments. J Biomed Mater Res B Appl Biomater. 2013;101(8):1387-92. https://doi.org/10.1002/jbm.b.32957

14. Pereira GK, Amaral M, Simoneti R, Rocha GC, Cesar PF, Valandro LF. Effect of grinding with diamond-disc and -bur on the mechanical behavior of a Y-TZP ceramic. J Mech Behav Biomed Mater. 2014;37:133-40. https://doi.org/10.1016/i.jmbbm.2014.05.010

15. Guilardi LF, Pereira GK, Gündel A, Rippe MP, Valandro LF. Surface micro-morphology, phase transformation, and mechanical reliability of ground and aged monolithic zirconia ceramic. J Mech Behav Biomed Mater. 2017;65:849-56. https://doi.org/10.1016/i.jmbbm.2016.10.008

16. Pereira GK, Fraga S, Montagner AF, Soares FZ, Kleverlaan CJ, Valandro LF. The effect of grinding on the mechanical behavior of Y-TZP ceramics: a systematic review and metaanalyses. J Mech Behav Biomed Mater. 2016;63:417-42. https://doi.org/10.1016/i.jmbbm.2016.06.028

17. Pereira GK, Silvestri T, Camargo R, Rippe MP, Amaral M, Kleverlaan $\mathrm{CJ}$ et al. Mechanical behavior of a Y-TZP ceramic for monolithic restorations: effect of grinding and lowtemperature aging. Mater Sci Eng C. 2016;63:70-7. https://doi.org/10.1016/i.msec.2016.02.049

18. Pereira GK, Silvestri T, Amaral M, Rippe MP, Kleverlaan CJ, Valandro LF. Fatigue limit of polycrystalline zirconium oxide ceramics: effect of grinding and low-temperature aging. J Mech Behav Biomed Mater. 2016;61:45-54. https://doi.org/10.1016/i.jmbbm.2016.01.006

19. Pereira GK, Venturini AB, Silvestri T, Dapieve KS, Montagner AF, Soares FZ et al. Low-temperature degradation of Y-TZP ceramics: A systematic review and metaanalysis. J Mech Behav Biomed Mater. 2015;55:151-63. https://doi.org/10.1016/j.jmbbm.2015.10.017

20. Garvie RC, Nicholson PS. Phase analysis in zirconia systems. J Am Ceram Soc. 1972;55(6):303-5. https://doi.org/10.1111/i.1151-2916.1972.tb11290.x

21. Toraya H, Yoshimura M, Somiya S. Calibration curve for quantitative analysis of the monoclinic tetragonal $\mathrm{ZrO} 2$ system by X-rays diffraction. J Am Ceram Soc. 1984;67(6):119-21. https://doi.org/10.1111/j.1151-2916.1984.tb19715.x

22. Kosmac T, Wagner R, Claussen N. X-Ray Determination of transformation depths in ceramics containing tetragonal ZrO2. J Am Ceram Soc. 1981;64(4):c72-3. https://doi.org/10.1111/j.1151-2916.1981.tb10285.x

23. Quinn GD. NIST recommended practice guide: fractography of ceramics and glasses. Gaithersburg: National Institute of Standards and Technology; 2007. 
24. Wachtman JB Jr, Capps W, Mandel J. Biaxial flexure tests of ceramic substrates. J Mater. 1972;7:188-94.

25. Weibull W. A statistical distribution function of wide applicability. J Appl Mech. 1951;18:293-7.

26. DIN ENV 843-5 Advanced technical ceramics: monolithic ceramics; mechanical tests at room temperature. Part 5: statistical analysis. Berlin: Deutsches Instut für Normung; 2007.

27. Jing Z, Ke Z, Yihong L, Zhiiian S. Effect of multistep processing technique on the formation of micro-defects and residual stresses in zirconia dental restorations. J Prosthodont. 2014;23(3):206-12. https://doi.org/10.1111/jopr.12094

28. Kosmac T, Oblak C, Jevnikar P, Funduk N, Marion L. The effect of surface grinding and sandblasting on flexural strength and reliability of Y-TZP zirconia ceramic. Dent Mater. 1999;15(6): 426-33. https://doi.org/10.1016/S0109-5641(99)00070-6

29. Kosmac T, Dakskobler A, Oblak Č, Jevnikar P. The strength and hydrothermal stability of Y-TZP ceramics for dental applications. Int J Appl Ceram Technol. 2007;4(2):164-74. https://doi.org/10.1111/j.1744-7402.2007.02124.x

30. Kosmac T, Oblak C, Marion L. The effects of dental grinding and sandblasting on ageing and fatigue behavior of dental zirconia (Y-TZP) ceramics. J Eur Ceram Soc. 2008;28(5): 1085-90. https://doi.org/10.1016/i.jeurceramsoc.2007.09.013

31. Işeri U, Ozkurt Z, Yalnız A, Kazazoğlu E. Comparison of different grinding procedures on the flexural strength of zirconia. J Prosthet Dent. 2012;107(5):309-15. https://doi.org/10.1016/S0022-3913(12)60081-X

32. Swain MV, Hannink RH. Metastability of the martensitic transformation in a $12 \mathrm{~mol} \%$ ceria-zirconia alloy: grinding studies. J Am Ceram Soc. 1989;72(8):1358-64. https://doi.org/10.1111/j.1151-2916.1989.tb07652.x

33. Karakoca S, Yilmaz $\mathrm{H}$. Influence of surface treatments on surface roughness, phase transformation, and biaxial flexural strength of Y-TZP ceramics. J Biomed Mater Res B Appl Biomater. 2009;91(2):930-7. https://doi.org/10.1002/jbm.b.31477

34. Kosmac T, Oblak C, Jevnikar P, Funduk N, Marion L. Strength and reliability of surface treated Y-TZP dental ceramics. J Biomed Mater Res. 2000;53(4):304-13. https://doi.org/10.1002/10974636(2000)53:4<304::AID-JBM4>3.0.CO;2-S

35. Işerı U, Ozkurt Z, Kazazoğlu E, Küçükoğlu D. Influence of grinding procedures on the flexural strength of zirconia ceramics. Braz Dent J. 2010;21(6):528-32. https://doi.org/10.1590/S0103-64402010000600008

36. Guazzato M, Quach L, Albakry M, Swain MV. Influence of surface and heat treatments on the flexural strength of Y-TZP dental ceramic. J Dent. 2005;33(1):9-18. https://doi.org/10.1016/i.jdent.2004.07.001

37. Papanagiotou HP, Morgano SM, Giordano RA, Pober R. In vitro evaluation of low-temperature aging effects and finishing procedures on the flexural strength and structural stability of Y-TZP dental ceramics. J Prosthet Dent. 2006;96(3):154-64. https://doi.org/10.1016/i.prosdent.2006.08.004

38. Pereira, G., Amaral, M., Cesar, P.F., Bottino, M.C, Kleverlaan, C.J., Valandro, L.F., $2015^{\mathrm{b}}$ Effect of low-temperature aging on the mechanical behavior of ground Y-TZP. J Mech Behav Biomed Mater. 2014;45:183-92. https://doi.org/10.1016/i.jmbbm.2014.12.009

39. Muñoz-Tabares JA, Jiménes-Piqué E, Reyes-Gasga J, Anglada M. Microstructural changes in ground 3Y-TZP and their effect on mechanical properties. Acta Mater. 2011;59(17): 6670-83. https://doi.org/10.1016/j.actamat.2011.07.024

40. Evans AG, Burlingame N, Drory M, Kriven WM. Martensitic transformations in zirconia particle size effects and toughening. Acta Metall. 1981;29(2):447-56. https://doi.org/10.1016/0001-6160(81)90170-X 